Objective: This study aims to evaluate circulating tumor cells isolated by high gradient magnetic separation of circulating endothelial cells (CECs) as a diagnostic tool for ovarian cancer. Methods: Thirty-six newly diagnosis with pelvic mass who were scheduled for surgery were included in this study. Blood samples were obtained from patients. CECs were identified with fluorescent microscopy by expert cytologists. The diagnostic performance of CECs was compared with the histopathological results.

Results: Definite diagnosis showed that twenty-six patients were later diagnosed as having malignant ovarian cancer (69.4\%), 5 cancerous patients (19.2\%) had metastatic ovarian tumor from gastrointestinal origin, and 10 patients were diagnosed as benign ovarian tumor (30.6\%). Serum from patients were extracted for CECs were found in 10 patients. CECs demonstrated modest diagnostic performance for malignant ovarian cancer with accuracy, sensitivity and specificity of $38.9 \%, 26.9 \%$, and $70.0 \%$, respectively.

Conclusion: CECs has low sensitivity. However, the clinical value of CECs in borderline ovarian tumor cases must be further investigated. This technique needs more development to improve sensitivity for diagnostic for ovarian cancer.

Poster (032)

Epithelial Ovarian Cancer including Borderline Tumor

https://doi.org/10.3802/jgo.2021.32.S1.032

\section{Knockdown of E2F4 suppresses the growth of ovarian cancer cells via cell cycle pathway}

\section{Young Tae Kim,," Eun Ji Nam, ${ }^{1}$ Sang Wun Kim,' Dae Woo Lee \\ 'Yonsei University College of Medicine, Seoul, Korea (ytkchoi@yuhs.ac) ${ }^{2}$ Catholic University College of Medicine, Seoul, Korea}

Objective: Ovarian cancer is one of the leading causes of death from gynecological cancer in the developed countries. The E2F family is a group of transcription factors that play decisive roles in the control of cell proliferation and cell cycle progression through the transcriptional activation of target genes in higher eukaryotes. However, the carcinogenic role of E2F transcription factor 4 (E2F4) remains unclear in ovarian cancer. In this study, we investigated the underlying molecular mechanism of E2F4 in human ovarian cancer cells (OCC).

Methods: The expression levels of E2F4 were demonstrated by quantitative real-time polymerase chain reaction (qRT-PCR). Small interfering RNA was used to suppress E2F4 expression. The effects of suppressing E2F4 on cell proliferation, migration and differentiation were evaluated by cell proliferation assay, wound healing assay and colony formation assay in vitro. The expression level of cyclins in OCC influenced by E2F4 was detected by Western blot.

Results: The expression of E2F4 mRNA and protein showed a significant higher level in OCC lines compared with controls. Depletion of E2F4 inhibited the cell proliferation and suppressed the cell migration and colony formation ability compared to control. Moreover, Western blot analysis showed that the expression of cyclin A, cyclin D and CDK2 was upregulated after the knockdown of E2F4.

Conclusion: Our study was consistent with the concept that E2F4 might contributed to proliferation and migration of OCCs by regulating the expression of cell cycle component in epithelial ovarian cancer.

Poster (O33)

Epithelial Ovarian Cancer including Borderline Tumor https://doi.org/10.3802/jgo.2021.32.\$1.033

\section{Multimodality active prehabilitation in patients undergoing surgery for epithelial ovarian cancer: a tertiary cancer center experience from Eastern India}

\author{
Upasana Palo," Jaydip Bhaumik, Anik Ghosh, Debapriya Mondal, \\ Jyoti B Lama, Zakaa Zawuaa
}

Tata Medical Center, Kolkata, India (upasana.obg@gmail.com)

Objective: This study aimed to determine if a multimodality active prehabilitation (MAP) program improves postoperative outcomes in patients undergoing surgery for epithelial ovarian cancer (EOC).

Methods: This was a prospective observational study over 6 months including 52 patients of EOC who underwent cytoreductive surgeries after MAP. These patients were compared with 51 patients with similar clinical characteristics at the same institution in the 6 months preceding the implementation of a MAP program (non-MAP group) by comparing frequency of complications with Clavien-Dindo (CD) score, median length of hospital stay, median time to chemotherapy, and 30-day-readmission rates.

Results: Twenty-nine patients (55.8\%) with interval debulking surgery, 13 with interval debulking surgery-hyperthermic intraperitoneal chemotherapy (25.0\%), 6 (11.5\%) with primary debulking surgery, 3 (5.8\%) with completion surgery, and 1 patient $(1.9 \%)$ with secondary cytoreduction were included in the MAP group ( $n=52$ ). $C D \geq 3$ complications were similar in the 2 groups. Median length of hospital stay was significantly shorter in the MAP group compared to the non-MAP group ( 8 vs. 10 days, $\mathrm{p}=0.006$ ). Median time to chemotherapy was significantly reduced ( 26 days vs. 30 days, $p=0.007$ ) and 30 -day- 
readmission rate was also significantly lower (1.9\% vs. $11.8 \%$, $\mathrm{p}=0.047)$ in the MAP group compared to the non-MAP group. Conclusion: MAP program appears to improve postoperative outcomes in patients with EOC undergoing surgery by shortening hospital stay, reducing time to chemotherapy, and lowering rates of hospital readmission. Larger prospective studies are warranted to better understand the role of prehabilitation in patients of EOC.

Poster (034)

Epithelial Ovarian Cancer including Borderline Tumor https://doi.org/10.3802/jgo.2021.32.S1.034

\section{Simplified selection criteria for the secondary cytoreductive surgery in recurrent ovarian cancer}

\section{Joo-Hyuk Son," Jimin Lee, Tae-Wook Kong, Jiheum Paek, Suk-Joon Chang, Hee-Sug Ryu}

Ajou University School of Medicine, Suwon, Korea (jh920@ajou.ac.kr)

Objective: Indications for secondary cytoreductive surgery (SCS) in ovarian cancer are often dependent on multiple confounding factors. We aimed to evaluate the treatment outcomes of recurrent ovarian cancer and investigate the factors for identifying patients who could most likely benefit from SCS. Methods: We retrospectively reviewed medical records of patients with recurrent ovarian cancer from 2003 to 2020. Treatment outcomes and potential factors influencing survival were evaluated between the patients who received chemotherapy alone (chemotherapy group) and those who received SCS (surgery group) after recurrence.

Results: Altogether, 262 patients with recurrent ovarian cancer were identified. The patients' median age was $53(20-80)$ years and $87.4 \%$ of patients had initial stage III/IV disease. Eightynine $(34 \%)$ patients received SCS. The median survival was $41.0(95 \%$ confidence interval $[\mathrm{CI}]=37.4-44.5)$ months, and 88.0 (95\% CI=64.2-111.7) months in the chemotherapy and the surgery groups, respectively. Patients in the surgery group were more likely to be young and showed no gross residual disease at primary surgery, BRCA $1 / 2$ mutation, limited metastatic lesion, and low rate of ascites $(\mathrm{p}=0.001, \mathrm{p}=0.001, \mathrm{p}=0.001, \mathrm{p}=0.001$, and $\mathrm{p}=0.04$, respectively). Multivariate analysis showed limited regional carcinomatosis (single region or up to 3 regions with limited carcinomatosis) ( $\mathrm{p}=0.045$ ) as the only significant factor for predicting no residual disease after SCS. In platinumsensitive recurrent patients with limited regional recurrence, the complete resection rate was $87.6 \%$.

Conclusion: SCS showed a significant survival impact on a well- selected patient population. Limited regional recurrence can be considered simplified criteria for SCS in platinum-sensitive recurrent ovarian cancer patients.

Poster (035)

Epithelial Ovarian Cancer including Borderline Tumor https://doi.org/10.3802/jgo.2021.32.S1.035

\section{The impact of appendectomy in grossly normal appendix on the survival outcomes among mucinous borderline ovarian tumors and mucinous ovarian carcinomas, in a tertiary hospital}

\author{
Kulisara Nanthamongkolkul, Krantarat Peeyananjarassri," \\ Ingporn Jiamset, Wuttipong Chusook, Sathana Boonyapipat, \\ Rakchai Buhachat \\ Prince of Songkla University, Songkhla, Thailand (krantarat@yahoo.com)
}

Objective: To evaluate the survival outcomes of appendectomy in grossly normal appendix among patients with mucinous borderline ovarian tumors and mucinous carcinomas Methods: A retrospective cohort study was performed in Songklanagarind Hospital between 2002 and December 2019. All patients with mucinous borderline ovarian tumors and mucinous ovarian carcinomas who had grossly normal appendix were included. Overall survival (OS) and progression-free survival (PFS) were analyzed using the Kaplan-Meier method and were compared with the log-rank test. Univariate and multivariate Cox regression analysis were used to determine the independent factors associated with OS and PFS.

Results: Of 534 patients, appendectomy was performed in 344 (64\%). Of these, $6(1.7 \%)$ were primary appendiceal tumors and $6(1.7 \%)$ were appendiceal metastasis from the ovarian tumors. During the median follow up of 74.5 months, 100 deaths were identified. The OS and PFS were better in patients with appendectomy compared to non-appendectomy (5year OS, $87.9 \%$ vs. $77.9 \%, p=0.001$ and 5 -year $\mathrm{PFS}, 81.2 \%$ vs. $62.6 \%, \mathrm{p}<0.001)$. Independent factors associated with worse OS and PFS were non-appendectomy, mucinous carcinoma, and advanced International Federation of Gynaecology and Obstetrics stage. Secondary ovarian tumor was the risk factor for a worse PFS but not significant in OS. Suboptimal debulking tumor also was the risk factor for a worse OS but not significant for PFS.

Conclusion: Appendectomy in grossly normal appendix among patients with mucinous borderline ovarian tumors and mucinous ovarian carcinomas was independent prognostic factor for a better OS and PFS. 